M. R. TREMBLAY* ET AL. (INFINITY PHARMACEUTICALS, CAMBRIDGE AND JOHNSON MATTHEY PHARMA SERVICES, DEVENS, USA; UNIVERSITY OF MONTREAL, CANADA) Development of a Multi Kilogram-Scale, Tandem Cyclopropanation Ring-Expansion Reaction en Route to Hedgehog Antagonist IPI-926

Org. Process Res. Dev. 2016, 20, 786-798.

\title{
Synthesis of IPI-926
}

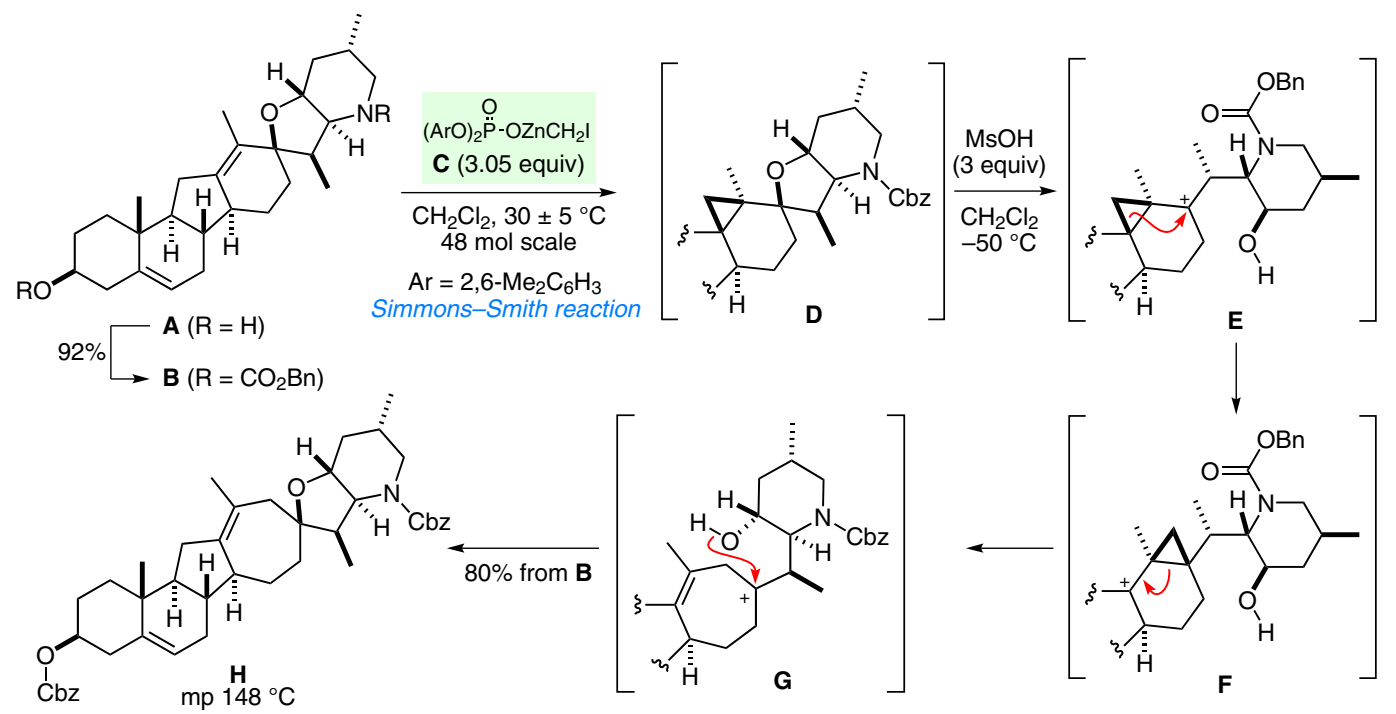

$$
5 \% \mathrm{Pd}(\mathrm{OH})_{2}
$$
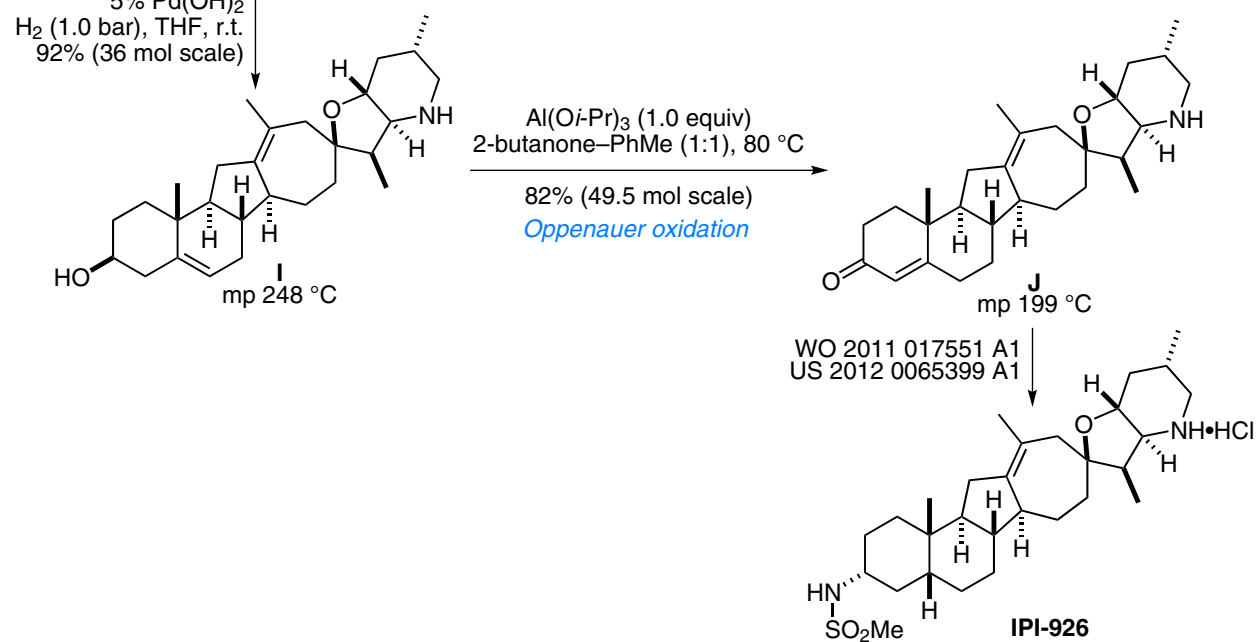

Significance: Cyclopamine $(\mathbf{A})$ is a teratogenic alkaloid isolated from the corn lily (Veratrum californicum). IPI-926 is a Hedgehog signalling pathway antagonist derived from cyclopamine that was evaluated for the treatment of cancer. The key step in the synthesis depicted is the robust and scalable Simmons-Smith cyclopropanation of $\mathbf{B}$ followed by an acid-catalyzed carbocation rearrangement.
Comment: For the large-scale Simmons-Smith reaction, a series of new safe and soluble iodomethylzinc bis(aryl)phosphate reagents (e.g., c) were prepared under mild conditions that were stable during the course of the reaction. Note the rare application of an Oppenauer oxidation $(\mathbf{I} \rightarrow \mathbf{J})$.

SYNFACTS Contributors: Philip Kocienski

Synfacts 2016, 12(07), 0657 Published online: 17.06.2016

Dol: 10.1055/s-0035-1562250; Reg-No.: K03316SF 